\title{
Associação entre fatores de risco cardiovascular e capacidade funcional de idosos longevos
}

\section{Association between cardiovascular risk factors and functional capacity of the oldest old}

Vanessa R. dos Santos ${ }^{1,2}$, Igor C. Gomes ${ }^{3}$, Lionai L. dos Santos ${ }^{2,4}$, Ricardo R. Agostinete ${ }^{2,4}$, Ismael F. Freitas Júnior ${ }^{1,2,4}$

\begin{abstract}
RESUMO
Modelo do estudo: Estudo transversal. Objetivo: Analisar a associação entre a presença de fatores de risco cardiovascular (FRC) e a capacidade funcional de idosos longevos. Materiais e Método: A amostra foi composta por 91 idosos com idade entre 80 e 90 anos (83,0 $\pm 2,3$ anos), sendo 60 mulheres $(82,9 \pm 2,1$ anos) e 31 homens (83,2 $\pm 2,6$ anos) residentes na cidade de Presidente Prudente/SP. Os FRC analisados foram: Hipertensão Arterial (HA) e excesso de gordura corporal (total e tronco). A presença de Hipertensão foi verificada por meio do questionário auto-referido baseado no Standard Health Questionnaire (SHQ). A avaliação da gordura corporal foi feita pela absorpiometria de dupla energia de raios-X (DEXA) e a capacidade funcional foi avaliada por meio dos testes funcionais (equilíbrio estático, velocidade usual de caminhada e força de membros inferiores). Para tratamento estatístico realizou-se o teste qui-quadrado, o software utilizado foi SPSS (13.0) e o nível de significância estabelecido foi de 5\%. Resultados: Os idosos com a presença de HA e excesso de \%GC apresentaram menor desempenho no teste de membros inferiores (83,3\% menor e 16,7\% maior), $p=0,011$ comparados aqueles com apenas um FCR. As idosas com a presença de HA e excesso de \%GTron também apresentaram menor desempenho no mesmo teste $(80,6 \%$ menor e $19,4 \%$ maior $), p=0,018$ e no teste de velocidade de caminhada $(80,6 \%$ menor e 19,4\% maior), $p=0,034$. Conclusão: A HA e o excesso de gordura corporal (total e tronco) agregados são FRC, que estão associados à redução da capacidade funcional de idosos longevos.
\end{abstract}

Palavras-chave: Composição Corporal. Hipertensão. Pressão Arterial Alta. Aptidão Física. Idoso de 80 Anos ou mais.

\section{Introdução}

O envelhecimento é um processo gradual, que promove alterações nos sistemas e no metabolismo humano. ${ }^{1,2}$ Apesar do processo de envelhecimento não estar, necessariamente, relacionado à ocorrência de fatores de risco cardiovascular (FRC), eles são frequentemente encontrados em idosos. ${ }^{3}$ São considerados FRC condições que predispõem um indivíduo a maior risco de desenvolver doenças do coração e dos vasos, ${ }^{4}$ e a presença dessas condições está diretamente relacionada à redução da capacidade funcional. ${ }^{5}$
1. Programa de Pós-Graduação em Ciências da Motricidade UNESP Rio Claro SP,

2. Centro de Estudos e Laboratório de Avaliação e Prescrição de Atividades Motoras (CELAPAM) - Presidente Prudente - SP,

3. Programa de Pós-Graduação em Epidemiologia - USP - São Paulo.

4. Departamento de Educação Física. UNESP- Presidente Prudente SP.
Correspondência:

Profa. Mda. Vanessa Ribeiro dos Santos Departamento de Educação física. Rua Roberto Simonsen, 305.

CEP: $19060-900$ - Presidente Prudente -SP Telefone: 1832295828 e-mail: van_vrs@yahoo.com.br

Artigo recebido em 1\%/07/2012 Aprovado para publicação em 19/12/2012 
A capacidade funcional (CF) representa a capacidade do indivíduo em realizar atividades físicas e motoras da vida diária sem dificuldades. ${ }^{6}$ Para idosos, a sua manutenção é muito importante, por proporcionar independência física para realizar as atividades cotidianas. ${ }^{7}$ Além disso, a capacidade funcional não está apenas associada a modificações clínicas ocasionadas pelo envelhecimento, mas também, pode ser utilizada para indicar complicações futuras relacionadas à saúde do idoso, tais como, incapacidade física, fragilidade, institucionalização e mortalidade. ${ }^{8}$

A associação entre a presença de FRC e alterações na funcionalidade ainda não são bem conhecidas. Em um idoso que apresente muitos FRC, a incapacidade de realização das atividades pode dever-se ao efeito de uma única condição ou a combinação de várias condições, e cada uma delas podendo afetar somente uma determinada atividade. ${ }^{5,9}$ Dessa forma, o objetivo do presente estudo foi analisar a associação entre a presença de FRC e a capacidade funcional de idosos longevos.

\section{Materiais e Método}

\section{Amostragem}

Estudo com delineamento transversal, conduzido no período de outubro de 2009 a maio de 2010 na cidade de Presidente Prudente (aproximadamente 210.000 habitantes),${ }^{10}$ localizada a oeste do Estado de São Paulo e com índice de desenvolvimento Humano (IDH) de 0,846 , sendo a $14^{\circ}$ do estado. ${ }^{11}$ De acordo com o Instituo Brasileiro de Geografia e Estatística ${ }^{12}$ a projeção para o ano de 2009 de brasileiros com idade igual ou acima de 80 anos era de 2.653.060, representado 1,1\% da população, partindo desse princípio a estimativa atual de idosos nessa faixa etária na cidade é de cerca de 2.100 habitantes. A secretaria municipal de saúde disponibilizou nome, endereço e telefone dos indivíduos com 80 anos ou mais. Com essas informações foi realizado sorteio de maneira aleatória dos 226 indivíduos e a abordagem inicial foi realizada por meio de telefone. Foram excluídos da amostra os indivíduos que não deambulavam, acamados, residentes da zona rural, institucionalizados, portadores de marca passo e que apresentavam dados incompletos em nosso banco de dados. Sendo assim, a amostra para o presente estudo foi composta por 123 indivíduos de ambos os sexos.

Os indivíduos convidados a participar do presente estudo foram esclarecidos sobre os objetivos e a metodologia empregada para a coleta dos dados. Somente os que assinaram o "Termo de Consentimento Livre e Esclarecido" fizeram parte da amostra. Todos os protocolos foram revisados e aprovados pelo Comitê de Ética em pesquisa da Universidade Estadual Paulista (Processo $n^{\circ}$ 26/2009). Ressalta-se ainda que todos os idosos participantes do estudo tiveram acesso aos resultados dos testes em que foram submetidos.

\section{Fatores de risco cardiovascular}

Os FRC analisados foram: a Hipertensão Arterial e o excesso de gordura corporal (total e tronco).

\section{Hipertensão Arterial}

A prevalência de Hipertensão da população em estudo foi obtida por meio do questionário de morbidades referidas, baseado no Standard Health Questionnaire for Washington State (SHQ). ${ }^{13}$ Trata-se de inquérito fechado, que analisa a presença/ ausência de doenças crônicas.

\section{Gordura Corporal}

Para análise da gordura corporal (total e tronco) foi utilizado o aparelho de Absortiometria de Raios-X de Dupla Energia (DEXA) da marca Lunar, modelo DPX-MD, software 4,7 que utiliza o modelo de três compartimentos (massa corporal magra, massa de gordura e mineral corporal). Esta técnica permite estimar a composição corporal total e por segmento. A medida é simples e necessita de auxílio mínimo do avaliado, que deve permanecer posicionado em decúbito dorsal, sem se movimentar no aparelho durante a realização da medida. Os resultados são transmitidos ao computador interligado ao aparelho e as variáveis referentes à gordura corporal são posteriormente analisadas. Os valores percentuais de gordura corporal total (\%GC) foi categorizado segundo a classificação de Morrow et al. ${ }^{14} \mathrm{e}$ do tronco (\%GTron) de acordo com os valores de mediana.

\section{Capacidade Funcional}

A CF foi analisada por meio dos testes de equilíbrio estático, velocidade usual de caminhada e força de membros inferiores presentes na seção $L$ do questionário SABE. ${ }^{15} \mathrm{O}$ teste de equilíbrio possui três etapas, realizadas em sequência (10 segundos cada): i) ficar em pé, com os pés unidos; ii) encostar o calcanhar de um dos pés na lateral do hálux do pé oposto, iii) ficar em pé, com pé na frente do outro. Os escores das três etapas foram somados e a partir da soma foi obtido o resultado final. 
No teste de caminhada o idoso tinha que caminhar por três metros na mesma velocidade utilizada para realizar as suas atividades cotidianas e o tempo do percurso foi cronometrado e registrado pelos avaliadores. Para avaliação da força de membros inferiores foi aplicado o teste de levantar/sentar da cadeira onde o idoso tinha que manter os braços cruzados sobre o peito, e ao sinal do avaliador ele tinha que levantar e sentar na cadeira, o mais rápido possível, cinco vezes em menos de 60 segundos. Os escores de cada teste variam entre 0 e 4 pontos. Para a classificação do desempenho dos idosos nos testes foram considerados: idosos com menor $\mathrm{CF}$ aqueles que obtiveram (0-2 pontos) e maior CF (3-4 pontos).

A avaliação da gordura corporal e aplicação do questionário de morbidade referida e dos testes funcionais foram realizadas por monitores treinados e capacitados do Centro de estudos e Laboratório de Avaliação e Prescrição de Atividade Motora (CELAPAM), do Departamento de Educação Física da Universidade Estadual Paulista, Campus de Presidente Prudente.

\section{Análise Estatística}

Para as variáveis numéricas, a normalidade do conjunto de dados analisado foi testada pelo teste de Shapiro-Wilk. A estatística descritiva foi composta por valores de mediana e intervalo interquartil. Os indivíduos foram distribuídos segundo o sexo e a comparação foi feita por meio do teste Mann-Whitney.

O teste qui-quadrado foi utilizado para testar a associação entre o desempenho nos testes de capacidade funcional e a presença dos fatores de risco. $\mathrm{O}$ tratamento estatístico foi realizado utilizando o software SPSS (SPSS inc. Chicago. IL), versão 13.0 e o nível de significância foi estabelecido em $5 \%$.

\section{Resultados}

A Tabela 1 apresenta as características gerais da amostra estudada, estratificada por sexo. Não houve diferença na idade dos idosos participantes do estudo. O grupo masculino apresentou valores de peso, estatura $(\mathrm{p}=0,001)$ significativamente maiores que o grupo feminino e a variáveis \%GC e \%GTron $(\mathrm{p}=0,000)$ foram maiores para o grupo feminino.

Na Tabela 2 são apresentados os valores percentuais da associação entre os FRC e o desempenho nos testes funcionais. Os idosos com a presença de HA e excesso de gordura (tronco ou total) agregados apresentaram menor desempenho no teste de força de membros inferiores $(p=0,002)$ e $(p=0,043)$ respectivamente. Não houve significância estatística entre os testes de equilíbrio e velocidade para caminhar, e a presença dos FRC.

No grupo masculino, idosos com a presença de HA e excesso de \%GC agregados apresentaram menor desempenho no teste de força de membros inferiores $(p=0,011)$. Não houve significância estatística entre os testes de equilíbrio e velocidade para caminhar, e a presença dos FRC Tabela 3.

No grupo feminino, idosas com a presença de HA e excesso de \%GTron agregados apresentaram menor desempenho nos testes de velocidade e força de membros inferiores $(\mathrm{p}=0,034)$ e $(\mathrm{p}=0,018)$, respectivamente Tabela 4.

\section{Tabela 1}

Características gerais da amostra e comparação segundo sexo

\begin{tabular}{lrrr}
\hline & \multicolumn{1}{c}{ Masculino } & Feminino & \\
\cline { 2 - 3 } Variáveis & Mediana (P25-P75) & Mediana (P25-P75) & 0,000 \\
\hline Peso (Kg) & $70,5(61,5-83,8)$ & $58,0(49,6-66,8)$ & 0,000 \\
Estatura (cm) & $165,5(159,5-169,3)$ & $150,5(145,7-154,8)$ & 0,765 \\
Idade (anos) & $82,8(81,5-84,1)$ & $82,7(81-84,8)$ & 0,000 \\
Gordura Tronco (\%) & $37,9(25,6-42,7)$ & $43,7(35,7-49,4)$ & 0,000 \\
Gordura Total (\%) & $32(23,5-35,6)$ & $42(35,4-47,3)$ & \\
\hline
\end{tabular}

P75= percentil 75; P25= percentil 25 


\section{Tabela 2}

Associação entre o desempenho nos testes funcionais e a presença de FRC cardiovascular de idosos longevos.

\section{DESEMPENHO NOS TESTES}

\begin{tabular}{|c|c|c|c|c|c|c|c|c|c|}
\hline \multirow[b]{3}{*}{ HA / \% GTron } & \\
\hline & \multicolumn{2}{|c|}{ Velocidade } & \multirow[b]{2}{*}{ p } & \multicolumn{2}{|c|}{ Equilíbrio } & \multirow[b]{2}{*}{ p } & \multicolumn{2}{|c|}{ Força MI } & \multirow[b]{2}{*}{$\mathbf{p}$} \\
\hline & Menor & Maior & & Menor & Maior & & Menor & Maior & \\
\hline Presença $=1$ & $60,0 \%$ & $40,0 \%$ & 0,290 & $30,0 \%$ & $70,0 \%$ & 0,735 & $52,5 \%$ & $47,5 \%$ & 0,002 \\
\hline Presença $=2$ & $70,6 \%$ & $29,4 \%$ & & $33,3 \%$ & $66,7 \%$ & & $82,4 \%$ & $17,6 \%$ & \\
\hline HA / \% GC & Menor & Maior & $\mathbf{p}$ & Menor & Maior & $\mathbf{p}$ & Menor & Maior & $\mathbf{p}$ \\
\hline Presença $=1$ & $61,3 \%$ & $38,7 \%$ & 0,420 & $38,7 \%$ & $61,3 \%$ & 0,420 & $54,8 \%$ & $45,2 \%$ & 0,043 \\
\hline Presença $=2$ & $69,4 \%$ & $30,6 \%$ & & $30,6 \%$ & $69,4 \%$ & & $75,0 \%$ & $25,0 \%$ & \\
\hline
\end{tabular}

$\mathrm{HA}=$ Hipertensão Arterial, \%GC= percentual de gordura corporal total, \%GTron= percentual de gordura do tronco Ml=membros inferiores, *presença $=1$ significa idosos com a presença de um dos FRC, presença=2 significa idosos com a presença dos dois FRC.

Tabela 3

Associação entre o desempenho nos testes funcionais e a presença de fatores de risco cardiovascular no grupo masculino.

\begin{tabular}{|c|c|c|c|c|c|c|c|c|c|}
\hline \multirow[b]{3}{*}{ HA / \%GTron } & \multicolumn{9}{|c|}{ DESEMPENHO NOS TESTES } \\
\hline & \multicolumn{2}{|c|}{ Velocidade } & \multirow[b]{2}{*}{$\mathbf{p}$} & \multicolumn{2}{|c|}{ Equilíbrio } & \multirow[b]{2}{*}{$\mathbf{p}$} & \multicolumn{2}{|c|}{ Força MI } & \multirow[b]{2}{*}{$\mathbf{p}$} \\
\hline & Menor & Maior & & Menor & Maior & & Menor & Maior & \\
\hline Presença $=1$ & $72,7 \%$ & $27,3 \%$ & 0,332 & $36,4 \%$ & $63,6 \%$ & 0,505 & $54,5 \%$ & $45,5 \%$ & 0,064 \\
\hline Presença $=2$ & $55,0 \%$ & $45,0 \%$ & & $25,0 \%$ & $75,0 \%$ & & $85,0 \%$ & $15,0 \%$ & \\
\hline HA / \% GC & Menor & Maior & $\mathbf{p}$ & Menor & Maior & p & Menor & Maior & p \\
\hline Presença $=1$ & $66,7 \%$ & $33,3 \%$ & 0,629 & $41,7 \%$ & $58,3 \%$ & 0,188 & $41,7 \%$ & $58,3 \%$ & 0,011 \\
\hline Presença $=2$ & $58,3 \%$ & $41,7 \%$ & & $20,8 \%$ & $79,2 \%$ & & $83,3 \%$ & $16,7 \%$ & \\
\hline
\end{tabular}

$\mathrm{HA}=$ Hipertensão Arterial, \%GC= percentual de gordura corporal total, \%GTron= percentual de gordura do tronco. Ml=membros inferiores, *presença $=1$ significa idosos com a presença de um dos FRC, presença=2 significa idosos com a presença dos dois FRC

\section{Tabela 4}

Associação entre o desempenho nos testes funcionais e a presença de fatores de risco cardiovascular no grupo feminino.

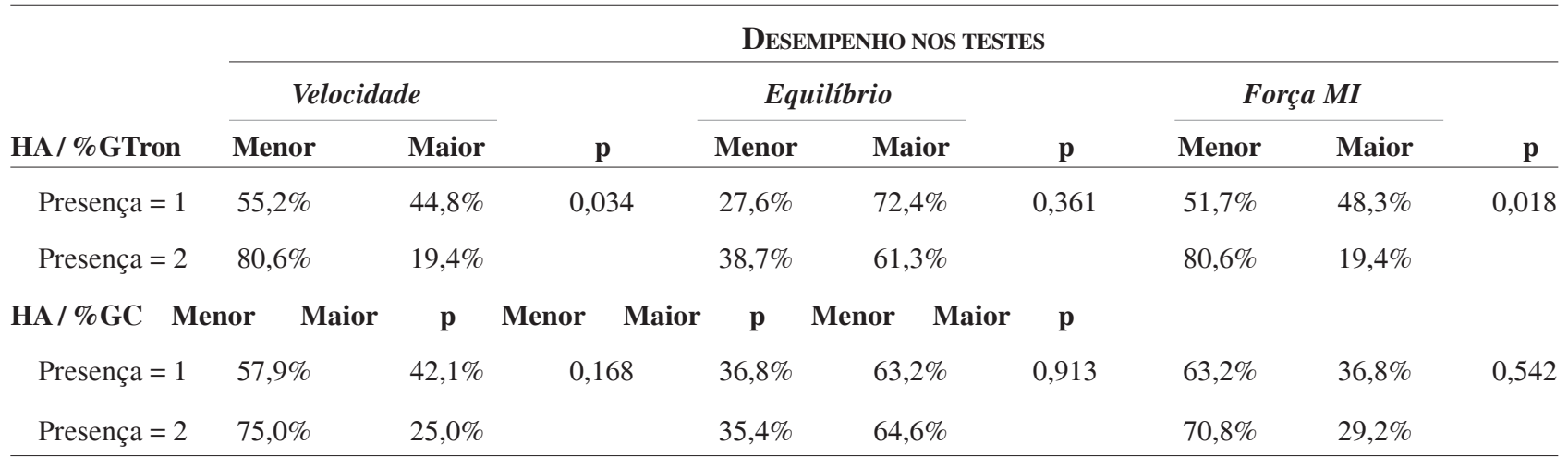

$\mathrm{HA}=$ Hipertensão Arterial, \%GC= percentual de gordura corporal total, \%GTron= percentual de gordura do tronco. Ml=membros inferiores, *presença $=1$ significa idosos com a presença de um dos FRC, presença=2 significa idosos com a presença dos dois FRC. 


\section{Discussão}

O presente estudo analisou a associação entre a presença de dois fatores de risco cardiovascular (Hipertensão e excesso de gordura corporal) e a capacidade funcional de idosos com idade igual ou superior a 80 anos, onde foi observado que, existe associação entre a presença desses fatores de risco agregados e a redução da capacidade funcional.

A hipertensão é um dos FRC mais prevalente entre os idosos brasileiros. Rigo et al ${ }^{16}$ encontraram uma prevalência de $84 \%$ em idosos da cidade de Novo Hamburgo/RS. No estudo conduzido por Gomes et al. ${ }^{3}$ a prevalência de HA em idosos com idade superior a 80 anos na cidade de Presidente Prudente-SP foi de $67,3 \%$. Esses achados são preocupantes, uma vez que, a hipertensão tem relação direta com o desenvolvimento de doenças cardiovasculares, ${ }^{17}$ bem como contribui com a redução da capacidade funcional. No estudo conduzido por Alves et al. (2007) na cidade de São Paulo foi observado que, idosos hipertensos aumentavam em $39 \%$ a chance de se tornarem dependentes funcionais. No presente estudo, a presença de HA agregada ao excesso de gordura corporal associou-se com pior capacidade funcional dos idosos longevos, do que um FRC isoladamente.

$\mathrm{O}$ excesso de gordura corporal é outro FRC que está associado à redução da capacidade funcional, ${ }^{7,18,19}$ bem como ao agravamento de doenças. ${ }^{20}$ Sallinen et al. ${ }^{21}$ identificaram associação negativa entre o \% GC e velocidade máxima de caminhada em indivíduos com idade superior a 55 anos. Uma das explicações para a associação negativa entre a gordura corporal e capacidade funcional deve-se ao fato de que, a maior quantidade de massa gorda ou maior $\%$ GC pode aumentar a sobrecarga corporal, limitando os movimentos e aumentando o estresse nas articulações e músculos, acentuando o risco de incapacidade nos idosos. ${ }^{22}$

$\mathrm{O}$ risco de incapacidade funcional é maior para mulheres com excesso de gordura do que para homens. ${ }^{23}$ Esse fato foi evidenciado no estudo de Rech et al. ${ }^{7}$ no qual foi observado uma relação inversa entre excesso de gordura corporal e capacidade funcional de idosas. Esses achados corroboram com os nossos resultados que, revelaram que o excesso de gordura agregada à presença de HA no grupo feminino exerceu maior influencia sobre a capacidade funcional comparado ao grupo masculino.

Em relação à distribuição da gordura corporal, a localizada na região abdominal, além de oferecer maior risco para eventos cardiovasculares, também parece ser mais prejudicial à capacidade funcional. No estudo conduzido por Bouchard et al. ${ }^{18}$ com idosos de ambos os sexos, e idade entre 68 e 82 anos, submetidos a testes funcionais, foi observado que o excesso de gordura abdominal foi mais prejudicial a capacidade funcional dos idosos do que a gordura corporal total ou acumulada em outra região. Esse fato corrobora com os nossos achados, uma vez que, idosas com excesso de gordura abdominal e presença de HA tiveram menor desempenho nos testes de velocidade de caminhada e força de membros inferiores. Com o avançar da idade o surgimento de FRC intensificam-se, ocasionando maior dependência nas atividades cotidianas. ${ }^{24,25}$ As mulheres tem uma expectativa de vida maior comparada aos homens, e apresentam maior incidência de FRC, demandando assim maior atenção à saúde. ${ }^{26}$

No estudo conduzido por Barbosa et al. ${ }^{27}$ não foi encontrada associação significativa entre o teste de levantar/sentar e excesso de \%GC em idosos mais jovens de ambos os sexos. Bouchard, Dionne e Brochu $^{18}$ investigaram 1.793 idosos canadenses de ambos os sexos e foi observado que homens com excesso de gordura tiveram menor desempenho no mesmo teste. Em nosso estudo, idosos longevos de ambos os sexos, com excesso de gordura (total ou abdominal) agregado à presença de HA apresentaram menor desempenho no teste. Barbosa et al. ${ }^{27}$ encontraram que, idosas com excesso de gordura apresentaram menor desempenho no teste de equilíbrio comparada as eutróficas, enquanto que, no grupo masculino não houve significância. Os autores Bouchard, Dionne e Brochu ${ }^{18}$ observaram essa evidência em idosos de ambos os sexos. No presente estudo não foi encontrada associação significativa entre o desempenho no teste e excesso de gordura em idosos longevos de ambos os sexos.

Uma das limitações do presente estudo foi o auto-referimento da Hipertensão, mas ressalta-se que esse tipo de procedimento é bastante utilizado em estudos populacionais devido a sua fácil aplicação, além disso, o delineamento transversal do estudo limita a capacidade de estabelecer relações de causalidade. Entretanto ressalta-se que ainda são escassos estudos que tiveram como objetivo verificar tais aspectos em idosos com idade superior a oitenta anos.

Em resumo, a HA e o excesso de gordura corporal (total ou tronco) agregados são FRC, que estão associados à redução da capacidade funcional de idosos longevos. 


\begin{abstract}
Study design: cross-sectional study. Objective: To assess the association between the presence of cardiovascular risk factor (CRP) and functional capacity of the oldest old. Methods: The sample 9onsisted of 91 elderly aged 80 and 90 years (83.0 \pm 2.5 years) with 60 women (82.2 \pm 2.1 years) and 31 men (83.2 $\pm 2,6$ years) residing in the city of Presidente Prudente - SP. The FRC were analyzed: arterial hypertension $(\mathrm{AH})$ and excess body fat (total and trunk). The presence of hypertension was verified by means of selfreported questionnaire based on the Standard Health Questionnaire (SHQ). Assessment of body was made by absorpiometria dual energy X-ray absorptiometry (DXA) and functional capacity was assessed by the functional tests (static balance, normal walking speed and force of the lower limbs). For statistical analysis we carried out the chi-square test, the software used was SPSS (13.0) and the significance level was set at $5 \%$. Results. In males, with hypertension and the presence of excess \%BF had lower performance in the lower limbs (83.3\% lower and $16.7 \%$ higher), $p=0.011$ compared to those with only a VCF. The elderly women with hypertension and the presence of excess GTron\% also had lower performance on the same test $(80.6 \%$ lower and $19.4 \%$ higher $), p=0.018$ and the test of walking speed $(80.6 \%$ lower and $19.4 \%$ higher), $p=0.034$. Conclusion: Arterial hypertension and excess body fat (total and trunk) aggregated are FRC, which are associated with reduced functional capacity of the oldest old.
\end{abstract}

Keywords: Body Composition. Hypertension. Blood Pressure, High. Physical Fitness. Aged, 80 and over.

\section{Referências}

1. Ferreira OG, Maciel SC, Silva AO, Santos WS, Moreira MA. O envelhecimento ativo sob o olhar de idosos funcionalmente independentes. Rev Esc Enferm USP. 2010; 44:1065-9.

2. North BJ, Sinclair DA. The Intersection between aging and cardiovascular disease. Circ Res. 2012;110:1097-108.

3. Gomes IC, Santos VR, Christofaro DG, Santos LL, Freitas Júnior IF. The most frequent cardiovascular risk factors in brazilian aged 80 years or older. J Appl Gerontol. December 26, 2011. Published online before print December 26, 2011, doi: 10.1177/0733464811427443. [2012 Abr 25].

4. Cooney MT, Dudina AL, Graham IM. Value and limitations of existing scores for the assessment of cardiovascular risk: a review for clinicians. J Am Coll Cardiol. 2009; 54: 1209-27.

5. Alves LC, Leimann BCQ, Vasconcelos MEL, Carvalho MS, Vasconcelos AGG, Fonseca TCO et al. A influência das doenças crônicas na capacidade funcional dos idosos do Município de São Paulo, Brasil. Cad Saúde Pública. 2007; 23:1924-30.

6. Arena R, Myers J, Williams MA, Gulati M, Kligfiel PJ, Balady GJ et al. Assessment of functional capacity in clinical and research settings: A scientific statement from the American Heart Association Committee on Exercise, Rehabilitation, and Prevention of the Council on Clinical Cardiology and the Council on Cardiovascular Nursing. Circulation. 2007;116: 32943.

7. Rech CR, Cruz JL, Araújo ED, Kalinowski FG, Dellagrana RA. Associação entre aptidão funcional e excesso de peso em mulheres idosas. Motricidade. 2010; 6: 47-53

8. Coelho CF, Burini RC. Atividade física para prevenção e tratamento das doenças crônicas não transmissíveis e da incapacidade funcional. Rev Nutr. 2009; 22:937-46.

9. Parahyba MI, Veras R. Diferenciais sociodemográficos no declínio funcional em mobilidade física entre os idosos no Brasil. Ciênc Saúde Coletiva. 2008; 13:1257-64.
10. IBGE - Instituto Brasileiro de Geografia e Estatística. Censo Demográfico e Contagem da População: População residente por sexo, situação e grupos de idade, 2010. Disponível em: www.sidra.ibge.gov.br. [Acessado: 25/03/2012].

11. IBGE - Instituto Brasileiro de Geografia e Estatística. Censo Demográfico e Contagem da População: População residente por sexo, situação e grupos de idade, 2000. Disponível em: www.sidra.ibge.gov.br. [Acessado: 25/03/2012].

12. IBGE - Instituto Brasileiro de Geografia e Estatística. Projeção da população do Brasil por sexo e idade para o período 1980-2050: revisão 2004. Rio de Janeiro: IBGE - Depis. Disponível em: http://www.ibge.gov.br. [Acessado: 25/03/2012]

13. Washington State Health Insurance Pool. Standard Health Questionnaire for Washington State. Washington; 2005.

14. Morrow JR, Jackson AW, Disch JG, Mood DP. Medida e avaliação do desempenho humano. 2nd: Artmed. 2003

15. Lebrão ML, Laurenti R. Saúde, bem-estar e envelhecimento: o estudo SABE no Município de São Paulo. Rev Bras Epidemiol. 2005; 8:127-45.

16. Rigo JC, Vieira JL, Dalacorte RR, Reichert CL. Prevalence of metabolic syndrome in an elderly community: comparison between three diagnostic methods. Arq Bras Cardiol. 2009; 93: 80-6.

17. Silva DA, Petroski EL. Associação entre diferentes proposições de pontos de corte para sobrepeso/obesidade e pressão arterial elevada em idosas. Rev Educ Fis. 2009; 20: 41522.

18. Bouchard DR., Dionne IJ, Brochu M. Sarcopenic/obesity and physical capacity in older men and women: Data From the Nutrition as a Determinant of Successful Aging (NuAge) the quebec longitudinal study. Obesity. 2009; 17: 2082-8.

19. Arnold AM, Newman AB, Cushman A, Ding J, Kritchevsky S. Body weight dynamics and their association with physical function, and mortality in older adults: the cardiovascular 
health study. J Gerontol. 2010; 65:63-70.

20. Kruger J, Ham SA, Prohaska TR. Behavioral risk factors associated with overweight and obesity among older adults: the 2005 National Health Interview Survey. Preventing Chronic Disease Public Health Research, Practice, and Policy 2009; 6: 1-17.

21. Sallinen J, Stenholm S, Rantanen T, Helioaara M, Sainio P, Koskinen S. Effect of age on the association between body fat percentage and maximal walking speed. J Nutr Health Aging. 2011; 15:427-32.

22. Bouchard DR, Choquette S, Dionne IJ, Brochu M. Is fat mass distribution related to impaired mobility in older men and women? Nutrition as a determinant of successful aging: the quebec longitudinal study. Exp Aging Res. 2011; 37:3, 34657.
23. Vincent HK, Vincent KR, Lamb KM. Obesity and mobility disability in the older adult. Obes Rev. 2010;11:568-79.

24. Fieller MM, Peres KG. Functional status and associated factors among the elderly in a southern Brazilian city: a populationbased study. Cad Saúde Pública. 2008; 24:40915.

25. Cardoso JH, Costa JS. Características epidemiológicas, capacidade funcional e fatores associados em idosos de um plano de saúde. Ciênc Saúde Coletiva. 2010; 15:2871-8.

26. Pedrazzi EC, Rodrigues RA, Schiaveto FV. Morbidade referida e capacidade funcional de idosos. Cienc Cuid Saude. 2007; 6:407-13.

27. Barbosa AR, Souza JMP, Lebrão ML, Marucci MFN. Estado nutricional e desempenho motor de idosos de São Paulo. Rev Assoc Med Bras.2007; 53:75-9. 